Nicola Rainisio, Marco Boffi, Eleonora Riva

\title{
6 Positive Change in Environment: Aesthetics, Environmental Flowability and Well-Being
}

\begin{abstract}
Environmental Psychology states that restoration and environmental preference could be explained by referring to our evolutionary past, or taking into account some of the universal features of the place. This contribution shows an alternative point of view, focusing on the concepts of flow. This chapter focuses on a new framework aiming to establish a link between optimal experiences, psychological well-being and aesthetic judgments, introducing the idea of Flowability as a subjective criterion to evaluate a place and consider it as potentially regenerative.
\end{abstract}

Keywords: Well-being; Restorativeness; Environmental preferences; Cultural selection; Evolutionary processes, Cultural artifacts; Flowability

\subsection{Introduction}

The connection between environmental features, aesthetic preference and psychological well-being is a paradigmatic example of the dilemmatic bond which links scientific psychology and common sense. It is in fact a widespread belief that such a link exists, and psychological research too has shown that human beings attend their favorite places as triggers of positive feelings and emotions when they are going through critical moments (Korpela et alii, 2001; 2003). The problem arises when this bond is investigated from a scientific point of view, considering that the theoretical analysis could be divided into at least three fundamental aspects. Firstly, it is necessary to examine whether there are distinctive environmental features that, more than others, elicit a positive response. The identification of these characteristics involves several higher order issues, such as, the assessment of whether these are universally recognized or automatically chosen. This query calls into question the second key issue, which concerns the subjective experience in dealing with these environmental features. To exert a preference means exercising a judgment based on available data, on the gestaltic qualities experienced in a specific context, or in an exercise of imagination/recalling of that event based on an environmental stimulus, such as in a video or a picture. The third aspect concerns the conceptualization, and the subsequent measurement, of the fundamental characteristics of psychological well-being. Also in this case it can be disputed whether the state of well-being has to be considered as a short or long term phenomenon, as when it occurs sharply when facing a specific environment. 
Since the seventies, environmental psychology has tried to give scientific foundation to such findings, already widely present in people's common sense. The mainstream explanation was oriented by an epistemology that considered the environment as a stimulus that triggers a normative human response or activates a cognitive scheme. Not surprisingly, studies of this kind have deepened only the first aspect, the one concerning environmental features that foster psychological well-being, exploring only in part the other two. Recently, however, some innovative proposals have been advanced taking into greater account the experiential (Ryan et alii, 2010; Joye \& van den Berg, 2011) and cultural aspects (Kitayama et alii, 2003; Buijs et alii, 2009) of our relationship with the environment. These theories suggest new contact points between the seminal works of Csikszentmihalyi (1975) and Kaplan \& Kaplan (1983), towards a new framework which integrates the complexity of the experience of place and its generative features (Rainisio \& Inghilleri, 2013) in the classical model. We suggest, in fact, that a phenomenological point of view would be useful to better define a systemic model of how the physical environment interacts with preference and psychological well-being, as it would be to include individual agency and emotionality. Before analyzing the more recent theories, it is necessary to deepen the mainstream framework and its underlying epistemological structure.

\subsection{Place as a Stimulus}

The models proposed in this field mainly follow two different ideas of mental functioning, in their turn deriving from two separate scientific paradigms. The first one is based on an evolutionary perspective, within which the interpretation of preference and psychological well-being is linked to the possibility that human beings have maintained in their aesthetic judgment a preference for habitats that maximize their chances of fitness, offering an abundance of resources and enlarging the opportunities for defense or protection. This perspective entails a corollary of at least three basic aspects. Firstly, the mechanism that activates preference and psychological well-being, resulting from phylogenesis, is automatic and not subject to the conscious control of the individual. Secondly, it follows that the subjective and cultural differences do not interfere with this automatic link, which is configured therefore as universal. Thirdly, people would tend to choose environments that have been a fertile ground for human evolution, and thus explains the data, shared by much research, which show a significant difference in preference between the natural and built environments, to all advantage of the first. A paradigmatic example is the savannah hypothesis (Orians, 1986). According to this theory the savannah, where allegedly the origin of man as a species took place, is also the prototype of the environment that guaranteed the hunter-gatherers of the Pleistocene the greater fitness possible.

The presence of scattered trees allowed to easily identify and collect fallen fruit and get find shelter from the sun, and at the same time constituted a minimum 
obstruction to the observation of the behavior of other predators, or to the search for a possible prey. The large animals present in the area guaranteed meat supply, while the grassy and wavy ground allowed orientation and the adoption of a nomadic lifestyle. This hypothesis is supported by evidences from various studies, mostly conducted in natural environment. Orians (1980), by analyzing natural drawings in a Japanese population, emphasized the tendency to modify the shapes of the local trees to make them look more like the savannah trees. Orians \& Heerwagen (1992) conducted a cross-cultural analysis of the preference for different types of trees, which resulted in shared patterns corresponding to the characteristics of the typical savannah vegetation. Similar results were reported by Summit \& Sommer (1999), which found a widespread preference for the acacia tree, and Lohr \& Pearson-Mims (2006), which showed more positive emotional reactions facing environments characterized by leafy trees compared to other shapes. Balling \& Falk (1982) have compared the preferences of a sample with respect to different biomes, including tropical forests, coniferous and temperate deserts and the savannah itself. The results showed a greater preference for savannah-like environments, however, limited to the lower age group. So the authors hypothesized that the savannah effect is predominant in childhood, but is later mitigated by individual and cultural inclinations. The data collected from this 1982 study have been re-used for a similar work (Falk \& Balling, 2010), based on the cross-cultural comparison between American and Nigerian citizens. The new conclusions identified the savannah biome as preferred by both populations, with no significant cultural differences. Appleton (1975) did not focus his prospect and refuge theory on a specific biome, but further underlined that our preference is oriented by our basic need to control the surrounding environment (prospect) and to be safe from external attacks by predators (refuge). This theory would explain our preference for panoramas and elevations, which at the same time provide control and protection allowing a broad view. A study conducted on a sample of children tested this hypothesis, resulting in only a partial confirmation, as the prospect factor was found to be closely related to aesthetic preference whereas the refuge factor did not have the same statistical significance (Fisher \& Shrout, 2006). Both theories follow the general idea that Wilson (1984) called biophilia, a supposed innate bond between human beings and the other living creatures developed in the phylogenesis as functional to the survival of the species.

Following an evolutionary approach, they also differ considerably from the models developed in a cognitive perspective. Cognitive science, focusing on skills (and limitations) of man as information processor, gives in fact less importance to the innate and automatic behaviors. It follows a model that explains the environmental preference through a steady process that matches the environmental stimuli and the subjective schemes of categorization and action. Hence arise two important corollaries. First of all, the matching activity is eminently subjective, as the content of the schemes differ from person to person. Secondly, the ability to cope with those stimuli would be largely influenced by the past experiences and, in a broader sense, by the 
culture of belonging. Schemes are in fact molded from the everyday experience and from the information and suggestions received in the educational process by caregivers and the community at large.

These aspects are an important innovation compared to the evolutionary model, as they propose an idea of subjective compatibility that goes beyond the phylogenetic determinism. Purcell (1986) suggested that preference might be given to those environments which possess an optimal level of discrepancy from one's own scheme, in order to generate a mild form of psycho-physical arousal without activating negative feelings such as boredom or anxiety. Conversely, Whitfield (1983) stated that preference might be oriented to environmental stimuli that mostly resemble the subjective scheme. Here too, as argued for other cognitivist theories, the representation of the relationship between man and environment appears to be "cold", unable to account for the emotional complexity of everyday experience. Moreover, even if a cognitive mediation is theorized, the holistic nature of that relationship is not taken into adequate consideration, focusing only on how subjective schemata are activated by external stimuli.

An integrative theory that unifies cognitive and evolutionary aspects was proposed by Kaplan, Kaplan \& Ryan (1998). The peculiarity of this point of view consists in considering cognition as the adaptive product of phylogenesis and assumes the existence of two basic needs for the optimal cognitive functioning, which is a prerequisite for survival, and specifically: the understanding of the context for action and the exploration of the same. If understanding is the central system that governs decision, exploration is equally important because it allows the acquisition of new information, and thus a qualitative increase in knowledge as a function of adaptation. The intersection of these two basic needs, understanding and exploration, with the degree of inference activated in the act of perception along the continuum of immediate-inferred, generates a $2 \times 2$ matrix which has four variables:

1. Coherence: comprehensibility immediately perceived (visual order)

2. Legibility: inferred comprehensibility (wayfinding, distinctive space)

3. Complexity: complexity immediately perceived (visual richness, high number of elements, various textures)

4. Mystery: complexity inferred (promise of new information on the environment deriving from its exploration)

While in the seminal study of Berlyne (1960) the complexity has been indicated as the main determinant of preference, in this theory the four factors described must remain in balance to maximize the pleasantness of the environment and perceived fitness with itself.

Despite the diversity of approaches, both the cognitive model on environmental aesthetics and the evolutionary models left two important aspects behind. The first concerns the subjective experience in dealing with environmental quality. The second deals with the short and long term effects of the exposure to restorative environments. 
It may therefore be useful to abandon the narrow frame of mainstream environmental psychology and engage in a reflection from a phenomenological point of view, which holistically reinserts those aspects that seem to be missing in the theories described above: the quality of the experience and the effects this may have on human behavior. According to this argument, a phenomenological framework should be discussed introducing two new epistemologies closely linked together, place as an experience and place as generative.

\subsection{Place as an Experience}

To integrate the mainstream framework with a phenomenological perspective could be useful to deepen the underestimated role of daily experience, since the basis of such an approach lies in the close ties between experience and place. Whereas phenomenology is the study of "things or experiences as human beings experience them" (Seamon, 1987), place is considered as a conditio sine qua non of all existing things, as for humans "to be is to be in a place" (Casey, 1993). People are bound by the body to an environmental context, and, consequently, to their experience. To experience is, in this perspective, mainly to experience in a situated body a situated thing. As Seamon (2013) pointed out:

Phenomenologically, place is not the physical environment separate from people associated with it but, rather, the indivisible, normally unnoticed phenomenon of person-or-people-experiencing-place (p. 11)

According to this point of view, he suggested (1982) that the human experience of the physical environment should be one of the main topics for environmental psychology. It is possible to refer to some scholars who have studied the environmental experiences and their relevance for aesthetic judgment and well-being. In popular culture the connection between environment and particular experiences, generally called peak experiences (Maslow, 1959), has been well-known since centuries; suffice it to mention the ritual vision quests of Native Americans, the spread of the romantic sublime in the European imaginary, or the centrality of the wilderness idea for the American culture. Moreover, nature is frequently associated with mysticism, ecstasy and other "supernatural" experiences in daily speech and thought. Kaplan \& Kaplan (1989) suggested that a complete restorative environment can be experienced as such when presenting four main features:

1. Fascination: to activate the involuntary attention permitting the voluntary one to recover.

2. Being away: to trigger feelings of being "elsewhere", far from the daily routine.

3. Extent: to experience the environment as unique and differentiated from others. 
4. Compatibility: to perceive a high fitness between the subjective goals and the possibilities offered by the socio-physical environment.

Restorative environments should then be conductive to a state of cognitive clarity, a state of mind characterized by the ability to focus on a cognitive task and to correctly select between the available opportunities.

Similarly, Williams \& Harvey (2001) described it as a transcendence experience, characterized by three factors:

1. a feeling of being overwhelmed and fascinated by the natural environment, with a strong involvement in the moment

2. a sense of strong novelty for the experience

3. a feeling of compatibility and ease

According to the authors, such an experience can also be divided in two distinct forms. A diminutive experience, characterized by a feeling of humility and insignificance, was found to be associated with high novelty and low compatibility. Conversely, the other typology is characterized by a strong sense of compatibility and familiarity.

A quite similar experience was described by Ryan and colleagues (2010) as triggered by the subjective vitality (Ryan \& Frederick, 1997). This study has emphasized that vitality and natural elements are related factors, assuming an experience of revitalization as main mediator between environment and psychological well-being, and also as being able to activate long-term positive processes on self-perception.

As biophilia could be indicated as the philosophical substratum for an evolutionary perspective, we suggest the flow theory (Csikszentmihalyi, 1975, 1990) as an integrative framework to resume the formerly described theories. In fact, the concept of optimal experience is characterized by some key elements that appear to integrate and summarize them:

a) Challenges-skills balance

Flow was found to be triggered by a perceived balance between situational challenge and personal skills, that is to say that the requests of the outer world (the environment) are balanced with the perceptions and the expectations coming from the inner world. This idea is very similar to the compatibility concept as formulated by Kaplan.

b) Merging

Flow was linked with a strong sensation of merging between the Self and the context, a feeling commonly reported by the researchers in environmental psychology, which could be summed up in the expression "deep experience".

c) Intrinsic motivation

During optimal experience, the main subjective motivation is the experience itself, the related sense of competence and completeness, without any external goal. A similar disinterested pleasure is considered as a main element of the aesthetic 
gratification in environment (e.g. Kant, 1790), that conversely is normally driven by an external object of attraction.

d) Effortless attention

During Flow the attentional resources are allocated voluntarily on a specific set of environmental stimuli, but this allocation is totally effortless and the control on action is perceived as automatic. Similarly, Kaplan defined fascination as a state of effortless attention, in which environmental stimuli are supposed to be able to attract the involuntary attention, permitting the voluntary one to recover.

e) Positive affects/clarity

Flow is characterized by a general sense of well-being and absence of boredom and anxiety, a sensation reported in relation to every transcendence experience in nature. Another characteristic that optimal experience shared with the concept of cognitive clarity is the peculiarity of being associated with feelings of full involvement, optimally functioning, mindfulness, and ideal exchange with the outer world.

As shown, the described theories have many points in common, but differ on a fundamental argument, specifically the causes of this experience and the role of attention. In Kaplan, the compatibility between man and environment is just one of the factors that lead to well-being, partially subjected to involuntary attraction of attention trough environmental features (fascination). His definition of well-being is then anchored to the concept of recovery, implying a return to a state of optimal cognitive functioning after a state of mental fatigue. The Flow Theory instead assigns a primary role to experience and not to the context, as the focus of attention is considered at the same time voluntary and effortless (Csikszentmihalyi \& Nakamura, 2010). Furthermore, well-being is not reached by a restoration process, but trough a feeling of growth of psychic complexity during the action. However, in both theories the balance between the environment and the internal world is considered as necessary to trigger the most intense feelings of well-being. In fact, Kaplan himself admits that involuntary attention can be attracted by what is being done and not only by environmental features (fascination for process), while Williams \& Harvey (2001) stated that the higher level of well-being is linked with the deep flow experiences, connoted by high levels of fascination and compatibility, and triggered by familiar contexts. Moreover, Flow Theory should also be a useful framework to explain the long term effects of positive experiences in environment and the mechanism of preference.

\subsection{Place as Generative}

According to the phenomenologists, place is not a static, but a dynamic and generative entity. It means that places and the experience thereof continuously evolve following six dynamic processes (Seamon, 2012): interaction, identity, release, realization, creation and intensification. This also means that significant changes may 
also be led by the relationship between person and place. Scholars have pointed out several findings in support of long-term changes associated to the experience of place. Kaplan \& Talbott (1983) stated that a wilderness experience had a deep impact on the individual personality of a group of young people involved in a natural camp. Wells (2000) found that a group of children living in an area with greater presence of trees developed a better ability to cope with stressful life events, also controlling the data for economic income. Kuo and colleagues (1998) underlined that the presence of natural areas empowered number and strength of social relationships in a Chicago apartment block.

Again, we suggest that an explanation to those findings should be found in flow theory and its developmental implications. First of all, after an experience of flow, the organization of the self is more complex than it had been before (Csikszentmihalyi, 1990, p.41). It means that an optimal experience may lead to a lasting change in self-perception and self-confidence. If there is no subjective experience without place, then every place compatible with a flow experience should become a positive place, and should be linked in memory and cognition with the positive experience of increased skills and self-esteem, especially if the context is relevant to that particular experience. It will depend on the subjective compatibility (or challenge-skills balance) as perceived in a precise moment of life. At the same time, the positive emotions experienced in a particular place could "broaden people's momentary thoughtaction repertories, widening the array of the thoughts and actions that come to mind" (Fredrickson, 2001) and promote, on the long-term, a complete mental health, a subjective state of flourishing in life (Keyes, 2002). According with this argument, place couldn't be only considered as a trigger for attentional and stress recovery, as argued by Kaplan \& Kaplan or Ulrich, but mainly as able to promote a long lasting well-being.

Moreover, as suggested by Csikszentmihalyi \& Robinson (1990) the objects involved in this experience (in our case, the place) will become subject of aesthetic pleasure and preference. In this perspective, a favorite place is where I lived the highest possible number of positive experiences. If we consider instead the typology of places, the favorite will be the one where I had the highest number of flow experiences or, the one that presents more evidence of the possibility of having experiences of flow based on past experience. According to these arguments, the flow experience could even be a trigger to support and strengthen place attachment and identification.

Lastly, all this will have a significant impact on places. In fact, a shared investment of psychic energy on a place will facilitate its conservation and protection, increase its semiotic complexity and activate its "transmission" to future generations, just as it happens for the other memes of our cultures (Dawkins, 1989; Inghilleri, 1999). Summarizing, it is not that evolutionary and stimulus-driven effects do not affect people's well-being in environment. These elements, rooted in our bio-cultural heritage, should be considered as positive factors to enable our optimal experiences in environment, which in turn activate several effects on psychological well-being. In 
the following section we will discuss some results that support these hypotheses, first providing experimental verification for our assumptions.

\subsection{Preliminary Results}

\subsubsection{Procedure}

An explorative research was conducted involving university students from Italy and Algeria ( $n=169)$. The main aim was to deepen the hypothesized links between environmental preference, restoration and flow experience. The procedure merged two consolidated research traditions in environmental psychology (restorativeness and preference). In the experiment, each student watched one picture of a landscape for a minute in a setting where potential outside disturbance was controlled for, after which a questionnaire was administered. In total, we showed nine pictures representing three types of biomes (savannah, forest and desert) and every picture was watched by at least 15 participants. The biomes have been selected because of their significance for both the environmental contexts and for the literature in this field. Our post-exposure questionnaire was composed of:

1. A short (18 items) version of Perceived Restorativeness Scale (PRS, Hartig et alii, 1997; Pasini \& Berto, 2007), including an item on environmental preference.

2. A short version of Revised Restoration Scale (RRS; Han, 2003).

3. An adapted version of Neighborhood Attachment Scale (NAS, Bonnes et alii; 1997), to deepen the role of place attachment.

The experiment was completed with a section dedicated to optimal experiences. The introductive part of the Flow Questionnaire (Csikszentmihalyi, 1975), which brings together some of the most common descriptions of the state of flow, was used as a stimulus to make participants understand what specific experience we were referring to.

That stimulus was followed by four ex novo items, designed to deepen the following aspects of flow experience in environment:

1. Perceived probability of a flow experience facing that environment ("Walking $a$. in a place like that I could feel this way").

2. Experiential past with regard to that environment ("It happened to me to feel this way in places like that").

3. Place capability in activating optimal experiences ("Places like that can help me to feel this way").

4. Strategic use of places as triggers for optimal experiences ("Sometimes I go to places like that to feel this way”). 
This scale is the first proposal of a Flow in Environment Scale (FLIES), an instrument able to analyze the connection between optimal experience and environmental contexts. The scale showed adequate psychometric properties.

The four items belong to one single factor, that we could call Environmental Flowability, intended as the perceived potential of a place to enable optimal experiences. This factor explains $79.74 \%$ of the variance, and has high saturation of items on the factor (all values higher than .84).

\subsubsection{Results}

We hypothesized that Flow would play a role in influencing preferences, wellbeing and restoration in environment, as well as that Flow should be linked with place attachment. Observing the matrix below (Tab.5.1) what emerges is a significant correlation between the perceived flowability, the two measures of restorativeness (PRS .698; RS .773), environmental preference (.656) and place attachment (.690).

Table 6.1: Matrix of correlations between flowability and other main variables

\begin{tabular}{lllll}
\hline & Preference & Restoration (PRS) & Restoration (RS) & Place attachment \\
\hline Flowability & .656 & .698 & .773 & .690 \\
N. & 166 & 159 & 130 & 160 \\
p. (2 tales) & .000 & .000 & .000 & .000 \\
\hline
\end{tabular}

Moreover, we investigated the influence of flowability on environmental preference by estimating its predictive potential through a linear regression. The results stated that the perceived flow opportunities explains the $43 \%$ of the preference variance (R. .656; R sq. .430; F. 123.57; P. .000), emphasizing the role of flow experience in producing aesthetic pleasure and influencing the judgment of preference. Figure 5.1 exemplifies this trend, showing a nearly-linear covariance between flowability and environmental preference. Also, we found similar and significant results about the flowabilityplace attachment links(R. .690; R sq. .476; F. 148.89; P. .000). This data indicated that almost half $(47,6 \%)$ of the variance in place attachment could be explained by flowability, providing confirmation to our hypothesis that identifies optimal experiences as important triggers to establish symbolic bonds between the person and the physical environment. On the other hand, considering the relationship between flowability and restorativeness (PRS and RRS), a different explanation is proposed. The strong correlation between the three scales and the high multicollinearity values (tolerance 
.394; VIF 2.537), could be due to a wide overlapping between the two constructs, whereas conceptualizations of flow and restoration tend to share some fundamental aspects as argued above. Not surprisingly, the higher FLIES-PRS correlations are observed on two PRS factors, fascination and compatibility, which call into question the two fundamental elements of interaction between the two: the optimal allocation of attentional resources and the compatibility between perceived challenges and environmental skills.

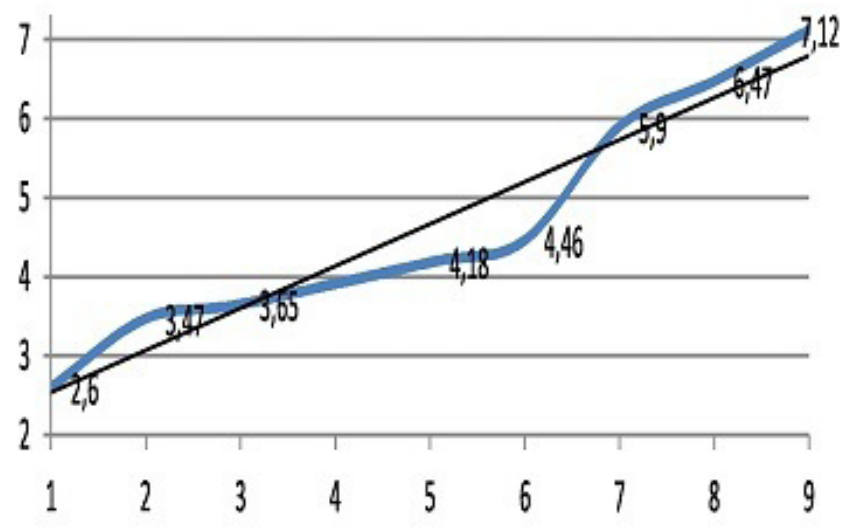

Flowability (mean)

\section{Preference}

Figure 6.1: Flowability-preference covariance

Pending further experimental support to this data, it can be suggested that the subjective perception of possible Flow experiences fosters aesthetic preference and influences place attachment. Also, a significant association was found between Flowability and restorativeness, assuming that the two constructs (Flow and restoration) could also be seen as two partially different descriptions of the same mental state (a peak experience), which could be adequately described by the notion of optimal experience as conceptualized by Csikszentmihalyi.

\subsection{Concluding Remarks}

The classical man-environment relationship model, based on an interaction between an external stimulus and a behavioral response, has so far offered a restricted explanation of the links between place, preference and subjective wellbeing. Environmental psychology has in fact privileged an evolutionary explanation based on an automatic response or, alternatively, on abstract information processing. However, we 
suggest the adoption of a phenomenological perspective and to consider place and experience as a totality which can only be understood through the study of subjective experience in an environment. In particular, we identified the flow experience as a framework able to explain environmental preference and the long term effects on subjective well-being. We also underlined that some existing theories, like Kaplan's Attention Restoration Theory, should be incorporated within this framework. Preliminary results showed a strong overlap between the two concepts, suggesting that people judge a place and its regenerative potential by relying on Flowability, namely evaluating its potential for generating optimal experiences.

\section{References}

Appleton, J. (1975). The experience of landscape. London, UK: Wiley.

Balling, J.D., Falk, J.H. (1982). Development of visual preference for natural environments. Environment and Behavior, 14, 5-28.

Berlyne, D.E. (1960). Conflict, arousal, and curiosity. New York, NY: McGraw-Hill.

Bonnes, M., Bonaiuto, M., Aiello, A., Perugini, M., Ercolani, P. (1997). A transactional perspective on residential satisfaction. In C. Despres, D. Piche (Eds.), Housing surveys: Advances in theory and methods (pp.75-99). Quebec, Canada: CRAD Universite Laval.

Buijs, A. E., Elands, B. H. M., Langers, F. (2009). No wilderness for immigrants: Cultural differences in images of nature and landscape preferences. Landscape and Urban Planning, 91 (3), 113-123.

Casey, E. S. (1993). Getting back into place. Bloomington, IN: Indiana University Press.

Csikszentmihalyi, M. (1975). Beyond Boredom and Anxiety, San Francisco: Jossey-Bass. Csikszentmihalyi, M. (1990). Flow. New York, NY: Harper \& Row.

Csikszentmihalyi, M., Nakamura, J. (2010). Effortless attention in everyday life: A systematic phenomenology. In B. Bruya (Ed.), Effortless attention: A new perspective in the cognitive science of attention and action (pp.179-190). Cambridge, MA: MIT Press.

Csikszentmihalyi, M., Robinson, R. E. (1990). The art of seeing. Malibu, CA: Getty Press. Dawkins, R. (1989). The selfish gene. London, UK: Oxford University Press.

Falk, J. H., Balling, J. D. (2010). Evolutionary influence on human landscape preference. Environment and Behavior, 42(4), 479-493.

Fisher, M.A., Shrout, P.E. (2006). Children's liking of landscape paintings as a function of their perception of prospect, refuge and hazard. Environment and Behavior, 38 (3), 373-393.

Fredrickson, B. L. (2001). The role of positive emotions in positive psychology: The broaden-andbuild theory of positive emotions. American psychologist, 56 (3), 218-226.

Han, K. T. (2003). A reliable and valid self-rating measure of the restorative quality of natural environments, Landscape \& Urban Planning, 995, 1-24.

Hartig, T., Korpela, K., Evans, G. W., Garling, T. (1997). A measure of restorative quality in environments. Scandinavian Housing \& Planning Research, 23, 3-26.

Inghilleri, P. (1999). From subjective experience to cultural change. Cambridge, UK: Cambridge University Press.

Joye, Y., van den Berg, A. (2011). Is love for green in our genes? A critical analysis of evolutionary assumptions in restorative environments research. Urban Forestry \& Urban Greening, 10, 261268.

Kant, I. (1987). Critique of Judgment. 1790. Trans. W. S. Pluhar. Indianapolis: Hackett. 
Kaplan, R., Kaplan, S. (1989). The experience of nature: A psychological perspective. New York, NY: Cambridge University Press.

Kaplan, R., Kaplan, S., Ryan, R.L. (1998). With people in mind: Design and management of everyday nature. Washington, DC: Island Press.

Kaplan, S., Kaplan, R. (1983). Cognition and environment: Functioning in an uncertain world. Ann Arbor, MI: Ulrich's.

Kaplan S., Talbot J.F. (1983). Psychological Benefits of a Wilderness Experience. In I. Altman, J. F. Wohlwill (Eds.) Behavior and the Natural Environment (163-203). New York, NY: Plenum Press.

Keyes, C. L. M. (2002). The mental health continuum: From languishing to flourishing in life. Journal of Health and Social Behavior, 43, 207-222.

Kitayama, S., Duffy, S., Kawamura, T., Larsen, J.T. (2003). Perceiving an object and its context in different cultures: A cultural look at the New Look. Psychological Science, 14, 201-206.

Korpela, K. M. (2003). Negative mood and adult place preference. Environment \& Behavior, 35 , 331-346.

Korpela, K. M., Hartig, T., Kaiser, F., Fuhrer, U. (2001). Restorative experience and self-regulation in favourite places. Environment \& Behavior, 33, 572-589.

Kuo, F. E., Sullivan,W. C., Coley, R. L., Brunson, L. (1998). Fertile ground for community: Innercity neighborhood common spaces. American Journal of Community Psychology, 26, 823-851.

Lohr, V.I., Pearson-Mims, C.H. (2006). Responses to scenes with spreading, rounded, and conical tree forms. Environment \& Behavior, 38, 667-688.

Maslow, A.H. (Ed.) (1959). New knowledge in human values. New York: Harper.

Orians. G.H. (1980). Habitat selection: general theory and applications to human behavior. In J. S. Lockard (Ed.), The Evolution of Human Social Behavior (pp. 49-66). Amsterdam: Elsevier.

Orians. G.H. (1986). An ecological and evolutionary approach to landscape aesthetics. In E. C Penning-Rowsell, D. Lowenthal (Eds.), Landscape meanings and values (pp. 3-22). London: Allen and Unwin.

Orians, G. H., Heerwagen, J. H. (1992). Evolved responses to landscapes. In J. Barkow, L. Cosmides \& J. Tooby (Eds.), The adapted mind (pp. 555-579). New York: Oxford University Press.

Pasini, M., Berth, R. (2007). Una scala per la misura della restorativeness dei luoghi. Quaderni DiPav, 20, 87-102.

Purcell, A. T. (1986). Environmental peception and affect: A schema discrepancy model. Environment \& Behavior, 18, 3-30.

Rainisio, N., Inghilleri, P. (2013). Culture, Environmental Psychology and Well-Being. In H. Knoop, A. Delle Fave (Eds.) Well-Being and Cultures (pp. 101-116). Netherlands: Springer.

Ryan, R.M, \& Frederick, C. (1997). On energy, personality, and health: Subjective vitality as a dynamic reflection of well-being. Journal of Personality, 65, 529-566.

Ryan, R.M., Weinstein, N., Bernstein, J.H., Brown K.W., Mistretta L., \& Gagne, M. (2010). Vitalizing effects of being outdoors and in nature. Journal of Environmental Psychology, 30 (2), 159-168.

Seamon, D. (1982). The phenomenological contribution to environmental psychology. Journal of environmental psychology, 2, 119-140.

Seamon, D. (1987). Phenomenology and environment-behavior research. In G. T. Moore and E. Zube (Eds.), Advances in environment, behavior and design, 1 (pp. 3-27). New York, NY: Plenum Press.

Seamon, D. (2013).Place attachment and phenomenology: The synergistic dynamism of place, In L. C. Manzo, P. Devine-Wright (Eds.), Place attachment (pp. 11-22). New York, NY: Routledge.

Summit, J., Sommer, R. (1999). Further studies of preferred tree shapes. Environment \& Behavior, 31, 550-556.

Wells, N. M. (2000). At home with nature: The effects of nearby nature on children's cognitive functioning. Environment \& Behavior, 32, 775-795. 
Whitfield, T.W.A. (1983). Predicting preference for familiar, everyday objects: An experimental confrontation between two theories of aesthetic behaviour. Journal of Environmental Psychology, 3, 221-237.

Williams, K., Harvey, D. (2001). Trascendent experience in forest environments. Journal of Environmental Psychology. 21, 249-260.

Wilson, E.O. (1984). Biophilia. Cambridge, MA: Harvard University Press. 